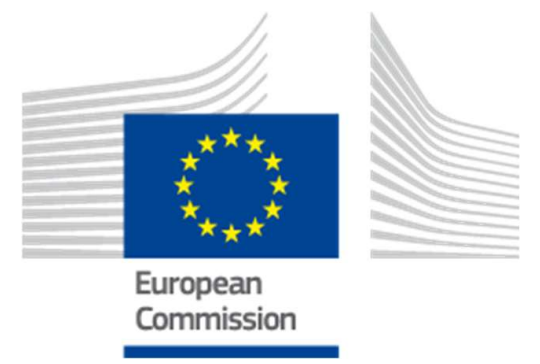

\begin{tabular}{|c|c|}
\hline Document Title & $\begin{array}{c}\text { Dimensioning a full color LED micro-display for } \\
\text { augmented reality headset in a very bright } \\
\text { environment }\end{array}$ \\
\hline Authors & $\begin{array}{l}\text { E. Quesnel }{ }^{1} \text {, A. Lagrange } 1 \text {, M. Vigier }{ }^{1}, \text { M. Consonni }{ }^{1} \text {, M. Tournaire } \\
\text { V. Le Marchand } \\
\text {, A. Suhm }\end{array}$ \\
\hline Issue date & March 6, 2019 \\
\hline Journal & Journal of the Society for Information Display. \\
\hline Doi & 10.1002/jsid.884 \\
\hline Acknowledgment & $\begin{array}{l}\text { This project has received funding from the Clean Sky } 2 \text { Joint } \\
\text { Undertaking under the European Union's Horizon } 2020 \text { research } \\
\text { and innovation programme under grant agreement No } 755497 .\end{array}$ \\
\hline Disclaimer & $\begin{array}{l}\text { The content of this article reflects only the author's view. } \\
\text { The Clean Sky Joint Undertaking is not responsible for any use that } \\
\text { may be made of the information it contains. }\end{array}$ \\
\hline
\end{tabular}




\section{Dimensioning a full color LED micro-display for augmented reality headset in a very bright environment}

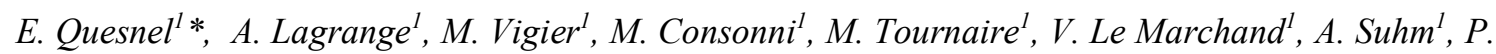

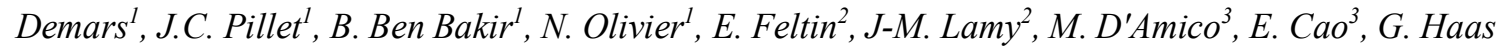
${ }^{4}$, L. Charrier ${ }^{4}$, P. Coni ${ }^{5}$.

${ }^{1}$ Université Grenoble-Alpes, CEA, LETI, MINATEC Campus, 17 rue des Martyrs, F-38054 Grenoble cedex, France.

${ }^{2}$ NOVAGAN, EPFL Innovation Park, 1015, Lausanne, Switzerland.

${ }^{3}$ NEXDOT, 102 avenue Gaston Roussel Biotech-bâtiment Pasteur, 93230, Romainville, France.

${ }^{4}$ MICROOLED, 7 Parvis Louis Néel, BP 50, BHT bâtiment, 38040 Grenoble cedex 09, France.

${ }^{5}$ THALES Avionics, 75 avenue Marcel Dassault, 33700 Merignac, France.

\section{ABSTRACT}

This paper focuses on the dimensioning of a very bright full color $10 \mu \mathrm{m}$-pitch Light Emitting Device (LED) micro-display for avionics application. Starting from the specifications of head mounted display to be used in an augmented reality optical system, a theoretical approach is proposed that enables predicting the specifications of the main technology building blocks entering into the micro-display manufacturing process flow. By taking into account various material and technological parameters, kept as realistic as possible, it is possible to assess the feasibility of a very bright LED micro-display $\left(1 \mathrm{Mcd} / \mathrm{m}^{2}\right.$ full white $)$ and to point out the main limitations. The theoretical specifications are then compared to the technical results obtained so far in the framework of the H2020 Clean Sky "HILICO" project. It shows that $350,000 \mathrm{~cd} / \mathrm{m}^{2}$ of white emission may be accessible with the present Gallium Nitride (GaN)-micro-LED technology provided a color conversion solution with stable external quantum efficiency of $30 \%$ is available. Beyond such level of luminance, the inherent limitations of driving circuit $(4 \mathrm{~V}, 15 \mu \mathrm{A}$ per pixel) commands working with materials enabling higher External Quantum Efficiency (EQE). In particular, $10 \mu \mathrm{m}$-pitch micro-LEDs with electroluminescence EQE of $15 \%$ and color conversion EQE approaching $60 \%$ are needed, opening the way to future challenging material and technology research developments.

\footnotetext{
* Contact person: etienne.quesnel@cea.fr
} 


\section{Introduction}

While projectors and cameras (viewfinders) have been dominating the micro-display market so far, the future market should be mainly driven by accelerating demand for micro-displays in Head Mounted Displays (HMD) and Head Up Display (HUD) applications. Today, the popular Augmented Reality (AR) headsets suffer from lack of luminance to allow the display of readable information against a very bright landscape, in particular for avionics or automotive use. Among available display technologies, best performing commercial products can reach over $20,000 \mathrm{~cd} / \mathrm{m}^{2}$ for monochrome, and over $3,000 \mathrm{~cd} / \mathrm{m}^{2}$ for high contrast bi-color OLED (Organic Light Emitting Device) - based micro-displays ${ }^{1}$ with recent evolutions toward a luminance of $10,000 \mathrm{~cd} / \mathrm{m}^{2}$ and full color thanks to improved materials and device architectures. In the same manner, recent full color micro- Liquid Cristal Displays $(\mathrm{LCD})^{2}$ are reported with at least $34,000 \mathrm{~cd} / \mathrm{m}^{2}$ of luminance, with the known downsides of such technologies, namely a poor black level and low power efficiency.

However, for some AR applications as in avionics or automotive, even brighter microdisplays are required, so there is a need for alternative micro-display technologies. With this respect, inorganic micro-LED displays are viewed as one of the most promising emerging solutions and has gained considerable attraction in recent years ${ }^{3,4}$. While the manufacturing of monochrome LED micro-displays have been demonstrated on several occasions by research and industry key players ${ }^{5,6,7}$ there is still a lack of high luminance full color microdisplay prototypes demonstrating red, green and blue (RGB) pixels integrated on the same display. In the present avionics HMD development, because of the necessary compactness of our projection system, it is precisely this last approach which needs to be explored.

The epitaxy of blue or green emitting GaN/InGaN epilayers is well controlled for years, however those of red emitting is still questionable because of the difficulty to grow very high In content-stable epilayers. Despite a very recent demonstration ${ }^{8}$, the native GaN/InGaN 
red color approach still requires further epitaxy studies to relax the internal stress in the epilayer. Hence, the best solution today for building a RGB LED micro-display is to start from an array of blue emitting pixel directly transferred and bonded to a silicon-based driving integrated circuit (IC) and partially covered with blue-to-green and blue-to-red

converters. This approach investigated by many research groups ${ }^{9,10,11}$ is usually based on the use of quantum dot (QD) nano-converters. Among the different issues encountered with such QD approach, one key issue still remains the insufficient blue light absorption ${ }^{10}$ incompatible with the need for pure color emission with no residual blue light component.

In this paper, our objective is to propose a methodology to specify step by step the different technology building blocks entering into the micro-display manufacturing process flow. Starting from the specifications of the display to be used in an avionics HMD application, an approach to specify the various building blocks is proposed and detailed. On this basis, technology and material specifications are deduced and compared to our technology capabilities. For that purpose, the capabilities of the technology building blocks developed within the HILICO European project ${ }^{12}$ are reviewed including the micro-display driving IC, the micro-LED technology and two potential color conversion routes including the QDs and the Quantum well (QW)-based converters.

\section{Micro-display application requirements:}

The development implemented in the HILICO project aims at displaying information and videos through the windshield of airplane cockpit (figure 1a). The sketch of figure 1b depicts the basic elements involved in the head mounted projection system. To achieve an acceptable user experience and help the pilot with an improved situational awareness, the displayed information shall be readable under the most severe daylight environment. SAE ARP 5288 standard demands a contrast greater than 1.2 under $34,000 \mathrm{~cd} / \mathrm{m}^{2}$ of background luminance 

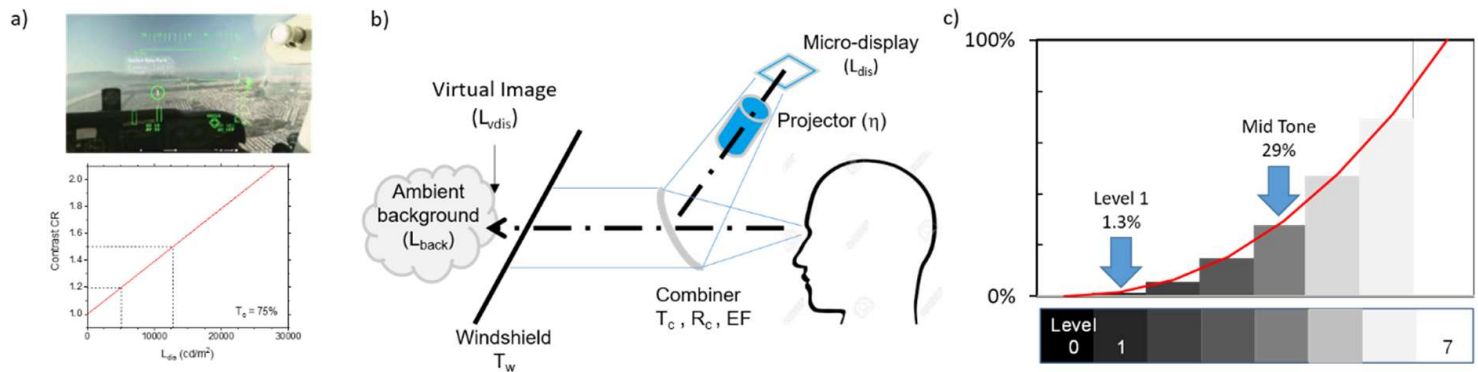

Figure 1: a) Typical scene observed by the HMD user with superposed virtual displayed image and dependence of virtual image contrast versus $L_{v d i s}$ assuming $L_{b a c k}=34,000 \mathrm{~cd} / \mathrm{m}^{2}$ and $T_{c}=75 \%$, b)Principle of an AR headset for avionics application; c) Grey scale and gamma curve (red). assuming a $100 \%$ transparent aircraft windshield $\left(\mathrm{T}_{\mathrm{w}}=1\right)$. This SAE standard defines the HUD Contrast Ratio (CR) as the measure of the ratio of the luminance of the virtual display $\mathrm{L}_{\mathrm{vdis}}$ relative to the real world background $\mathrm{L}_{\text {back. }}$ HMD display contrast ratio is thus defined as:

$$
\mathrm{CR}=\frac{L_{v d i s}+T_{c} L_{\text {back }}}{T_{c} L_{\text {back }}}
$$

$L_{v d i s}$ is the luminance of the virtual image collimated to infinity and seen by the HMD observer. $\mathrm{T}_{\mathrm{c}}$ is the optical transmission of combiner $\left(\mathrm{T}_{\mathrm{c}}=75 \%\right)$. This contrast value increases linearly with $\mathrm{L}_{\mathrm{vdis}}$ (figure 1a). To reach $\mathrm{CR}=1.2, L_{\text {vdis }}$ of $5,000 \mathrm{~cd} / \mathrm{m}^{2}$ is needed, but for a better user experience, a contrast of 1.5 corresponding to $L_{v d i s}=12,750 \mathrm{~cd} / \mathrm{m}^{2}$ is targeted. This requirement is for symbols at maximum luminance for a monochrome display (Green) without taking into account grey levels.

However, if we want to display a modern user interface, readable grey levels are needed, with a minimum contrast of 1.2 corresponding to $5,000 \mathrm{~cd} / \mathrm{m}^{2}$. Grey level scale is not linear and follows the well-known gamma curve:

$$
L_{v d i s}=L_{v d i s}^{\max } *\left[\frac{\text { GreyLevel }}{\text { GreyLevelMax }}\right]^{\gamma}
$$

The expression in brackets is the gamma scale, which graphical representation is given in figure $1 \mathrm{c}$ for $\gamma=2.2$ (video image) and eight levels of grey (level 0 for the black background, level 7 for the maximum luminance). In these conditions, mid tone input (level 4) corresponds to 29\% of the maximum luminance, and level 1 to $1.3 \%$. Hence, a readable level 1 should imply a 
maximum luminance $L_{v d i s}^{\max } \sim 385,000 \mathrm{~cd} / \mathrm{m}^{2}(5,000 / 0.013)$. This value is not only dangerous for human eye but also unreachable in a reasonable size and power consumption for a wearable AR device.

Actually, it is not reasonable to consider that level 1 is readable when superimposed with a bright outside world. Usual way to make the low levels of grey readable consists of adding a uniform luminance background to the displayed image. The drawback is that the intrinsic contrast of the displayed image is made lower, but the benefit is that the user can see the darkest details. Based on human factors studies and operational return from pilots, one can consider $L_{v d i s}^{\max } 20,000 \mathrm{~cd} / \mathrm{m}^{2}$ to be a reasonable level of maximum luminance. This is a good compromise between pilot visual comfort and preservation of grey scale perception by keeping readable several levels of grey.

As shown in figure $1 \mathrm{~b}$, the light produced by the micro-display $\left(L_{d i s}\right)$ propagates through the optics of projector and combiner. Global optical efficiency depends on transmittance and reflectance of the combiner $\left(T_{c}, R_{c}\right)$, transmittance of the windshield $\left(T_{w}\right)$ and efficiency of the projection optics $(\eta)$. We can write:

$$
L_{d i s}=\frac{E F}{\eta \cdot R_{c}} \cdot L_{v d i s}
$$

where EF is the expansion factor of the exit pupil expander. EF is strongly dependent on combiner design and can range from 1 with a classical combiner (without pupil expansion) to 10 with a more sophisticated one (with a waveguide and exit pupil expansion) designed for optimum user experience. Taking $\mathrm{R}_{\mathrm{c}}=25 \%, \eta=80 \%$ and $L_{v d i s}^{\max } \sim 20,000 \mathrm{~cd} / \mathrm{m}^{2}$ leads to a range of maximum micro-display luminance $L_{d i s}^{\max }=100,000$ to $1,000,000 \mathrm{~cd} / \mathrm{m}^{2}$.

This ultimate $1 \mathrm{Mcd} / \mathrm{m}^{2}$ luminance specification is clearly very challenging and will be very demanding in terms of technology control and quality of materials to be assembled. As already said, commercially available full color micro-display technologies involving LCD or OLED cannot exceed $\sim 30-40,000 \mathrm{~cd} / \mathrm{m}^{2}$. In return, inorganic LED as a promising emerging technology 
may pretend reaching much higher luminance. At CEA-Leti ${ }^{13}$, very high luminance values of 1(10) $\mathrm{Mcd} / \mathrm{m}^{2}$ have indeed been demonstrated on blue (green) passive displays. More recently ${ }^{6}$, functional monochrome LED micro-displays have demonstrated up to $10,000 \mathrm{~cd} / \mathrm{m}^{2}$ in green. On this technological basis and experience, the purpose of the next section will be to specify the materials and technologies required for $1 \mathrm{Mcd} / \mathrm{cm}^{2}$ and to assess the feasibility of such full color micro-display.

\section{Dimensioning the micro-display building blocks}

The previous specifications give the maximum luminance $\left(1 \mathrm{Mcd} / \mathrm{m}^{2}\right)$ that each white pixel needs to provide. To achieve such high luminance and as shown in figure 2, each white pixel is designed according to a 4 pixel architecture, one blue (B), one green (G) and two reds (R) arranged in a four pixel array with a pitch of $9.5 \mu \mathrm{m}$ and a micro-LED pixel size of $8 \mathrm{x} 8 \mu \mathrm{m}^{2}$. This RRGB arrangement of pixel array is chosen to limit and better balance the blue radiant powers to be produced at the level of the different micro-LEDs (see table I). As a result, while the white light will be produced by a $19 \times 19 \mu \mathrm{m}^{2}$ QUAD pixel, the R, G, B light components will be produced by each elementary pixel of the QUAD. At the display level the area to be considered for the elementary emitting pixel is actually $\mathrm{a}=9.5 \times 9.5 \mu \mathrm{m}^{2}$ a bit larger than the micro-LED size.

The RGB display technology chosen here involves 3 main technological levels involving the active matrix (silicon-based control IC) with the successive stacking on top of it of the microLED array and the conversion layers. The active matrix is a silicon-based integrated circuits (IC) used to drive the pixels of the micro-LED array and to monitor the video flux. The 3-level stacking requires sub-micron alignment accuracy, hence this operation is realized at 8-inch wafer level on a microelectronics manufacturing line. More details are given in section 5. 
At the level of each elementary pixel, the active matrix delivers the electrical power (ILED $\mathrm{x}$ $V_{\text {LED }}$ necessary to supply the micro-LED diode and emit the desired blue radiant power. The blue light is emitted by the pixelated $\mathrm{GaN} / \mathrm{InGaN}$ epilayer transferred on the active matrix. It is characterized by its electro-luminescence External Quantum Efficiency (EQE), noted $\eta_{E L}$, which depends on various parameters like the epilayer quality based on its density of dislocations and active structures, in particular. It is also dependent on the micro-LED array process including the pixel etching and pixel wall passivation, for instance. As for the emission of green and red light, while we are working with a monolithic blue epilayer, an additional color conversion layer is requested on top of it. This conversion layer is characterized by its photoluminescence EQE, noted $\eta_{P L}$.

On the basis of this technological approach our purpose is now to calculate the specifications of the various micro-display building blocks taking into account realistic material conversion efficiencies $\eta_{E L}$ and $\eta_{P L}$. The building block specifications (see figure 2) include (i) at the level of the conversion layer, the color radiant power $\mathrm{p}_{\mathrm{B}}, \mathrm{p}_{\mathrm{G}}$ and $\mathrm{p}_{\mathrm{R}}$ to be produced at the exit of each corresponding pixel to reach a white luminance of $1 \mathrm{Mcd} / \mathrm{m}^{2}$, (ii) at the level of the micro-LED array, the blue radiant power to be converted $\mathrm{p}_{\mathrm{B}}{ }^{\mathrm{G}}$ and $\mathrm{p}_{\mathrm{B}}{ }^{\mathrm{R}}$ and (iii) at the level of the active matrix, the current $\left(\mathrm{I}_{\mathrm{LED}}\right)$ and bias voltage $\left(\mathrm{V}_{\mathrm{LED}}\right)$ to be delivered in each pixel. Two scenarios

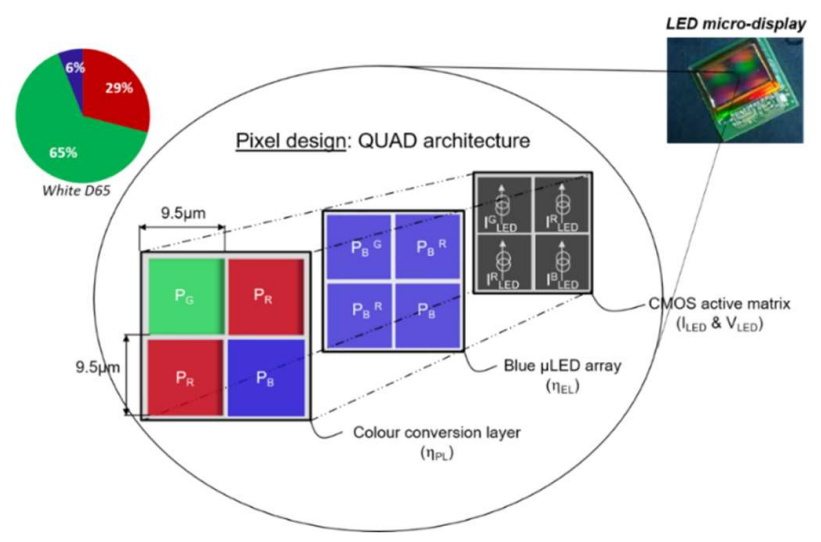

Figure 2: Sketch depicting the design of white pixel based on a 4-pixel architecture and the 3 levels of technology necessary to achieve it. The main parameters to take into account are indicated. The photometric pareto for D65 standard white light is inserted left side. 
will be considered with conversion efficiency values inspired from our own material experience and from literature data. The specifications deduced by calculation are summarized in table I. The first calculation must estimate the radiant power to be emitted by the white QUAD pixel. For that purpose, we consider a $1 \mathrm{Mcd} / \mathrm{m}^{2}$ white light having the colorimetric coordinates in the color space chromaticity diagram of a standard D65 white light (correlated color temperature of $6500 \mathrm{~K})$. In that case, the luminance photometric breakdown in red, green and blue components is estimated to be $29 \%$ red, $65 \%$ green and $6 \%$ blue (photometric pareto). That means that to get $1 \mathrm{Mcd} / \mathrm{m}^{2}$ white light, we need to generate the following color luminance values: $L_{\text {color }}=290,000 ; 650,000$ and $60,000 \mathrm{~cd} / \mathrm{m}^{2}$, for red $\left(\lambda_{R}=635 \mathrm{~nm}\right)$, green $\left(\lambda_{G}=525 \mathrm{~nm}\right)$ and blue $\left(\lambda_{B}=460 \mathrm{~nm}\right)$ color, respectively. Conversion of this luminance values into radiant power ( $\mathrm{P}_{\text {color }}^{W}$ : $\mathrm{P}_{\mathrm{R}}, \mathrm{P}_{\mathrm{G}}$ and $\mathrm{P}_{\mathrm{B}}$ ) can be done combining the following equations (4) and (5) knowing that in photometry:

$$
\mathrm{P}_{\text {color }}^{\mathrm{W}}=\frac{\mathrm{P}_{\text {color }}^{\mathrm{lm}}}{\left(683^{*} \mathrm{~V}(\lambda)\right)}
$$

Where $P_{\text {color }}^{W}$ and $P_{\text {colour }}^{l m}$ are the radiant power in watt and luminous power in lumen, respectively. $P_{\text {colour }}^{l m}$ is the luminous power actually seen by an observer and $\mathrm{V}(\lambda)$ is the luminous efficiency function for the photopic observer. These values are tabulated in the CIE 086-1990 standard. We take $\mathrm{V}(460)=0.06, \mathrm{~V}(525)=0.78$ and $\mathrm{V}(635)=0.22$.

If we assume a Lambertian pixel emission, $P_{\text {colour }}^{l m}$ is linked to the luminance $L_{\text {colour }}$ by the following relation:

$$
\mathrm{P}_{\text {color }}^{\operatorname{lm}}=\iint_{0}^{2 \pi s r} L(\cos \theta)^{n} \cdot d \Omega=\frac{2 \pi}{n+1} L_{\text {color }}=\pi L_{\text {color }}
$$

In that case the luminous intensity L(cd) has an angular distribution with follows a cosine law $\mathrm{L}=\mathrm{L}_{0} \cos ^{\mathrm{n}}(\theta)$ with $\mathrm{n}=1$.

Combining (4) and (5) gives finally:

$$
\mathrm{P}_{\text {color }}^{\mathrm{W}}=\frac{\pi \mathrm{L}_{\text {color }}}{683 . \mathrm{V}(\lambda)} \cdot \mathrm{A}
$$


Where $A$ is the emitting surface of the white QUAD pixel $\left(A=19 \times 19 \mu \mathrm{m}^{2}\right)$.

In practice, this color radiant power will be produced by one elementary color pixel of the QUAD after conversion of the blue light emitted by the diode of the micro-LED array. The blue radiant power $\mathrm{P}_{\mathrm{B}}^{\text {color }}$ (i.e; $\mathrm{P}_{\mathrm{B}}{ }^{\mathrm{R}}$ or $\mathrm{P}_{\mathrm{B}}{ }^{\mathrm{G}}$ in figure 2) necessary to produce the expected $\mathrm{P}_{\text {color }}^{\mathrm{W}}$ will depend on the photoluminescence conversion efficiency and we have:

$$
\mathrm{P}_{\mathrm{B}}^{\text {color }}=\frac{\mathrm{P}_{\text {color }}^{\mathrm{W}}}{\eta_{\mathrm{PL}}} \frac{\lambda_{\text {color }}}{\lambda_{\mathrm{B}}}
$$

where $\lambda_{\text {color }}=\lambda_{\mathrm{R}}$ or $\lambda_{\mathrm{G}}$.

Finally, knowing $\mathrm{P}_{\mathrm{B}}^{\text {color }}$ and the electroluminescence efficiency $\eta_{\mathrm{EL}}$ of the blue micro-LED we can deduce the current to be injected in the blue elementary pixel for a given bias voltage:

$$
\mathrm{I}_{\mathrm{LED}}^{\text {color }}=\frac{\mathrm{P}_{\mathrm{B}}^{\text {color }} \cdot 460}{\eta_{\mathrm{EL}} \cdot 1240}
$$

In table I, we have reported the values of $\mathrm{P}_{\text {color }}^{\mathrm{W}}, \mathrm{P}_{\mathrm{B}}^{\text {color }}$ and $\mathrm{I}_{\mathrm{LED}}^{\text {color }}$ calculated with equations (6), (7) and (8), respectively, for two scenarios. Scenario\#1 is the high scenario with materials exhibiting high EQE; scenario\#2 is the low scenario with lower EQE materials hence higher electric power. Note that $\mathrm{V}_{\mathrm{LED}}$ is arbitrary chosen to match the I/V characteristics of current micro-LED diodes.

We observe that for the green and red pixels, the blue micro-LEDs must emit up to 2.6 and 5.2 $\mu \mathrm{W}$ depending on scenario \#1 or \#2. That means up to 2.9 and $5.7 \mathrm{~W} / \mathrm{cm}^{2}$ of blue light maximum. This calculation gives an indication on the rather high optical power density of blue light that the conversion layers will have to cope with in a real high luminance micro-LED display application. As a comparison, in a LCD display emitting typically $1000 \mathrm{~cd} / \mathrm{m}^{2}$ D65 standard white light with a global optical conversion efficiency of $\sim 2 \%(50 \%$ for backlight color conversion and $4 \%$ for the LC panel integrating color filters) the blue optical power density is estimated to be two orders of magnitude lower, around $20 \mathrm{~mW} / \mathrm{cm}^{2}$. In the same manner, the data of table I give interesting indications on the specifications for the active matrix. In scenario 
$\# 1$, the $\mathrm{I}_{\mathrm{LED}} / \mathrm{V}_{\mathrm{LED}}$ characteristics seem rather reasonable with respect to expected IC performances (see section 4). In return, in scenario \#2 with material conversion efficiencies divided by 2 , the ILED/VLED characteristics could rapidly exceed the driving IC capacities.

\begin{tabular}{|c|c|c|c|c|c|c|c|c|c|c|}
\hline & \multicolumn{4}{|c|}{ QUAD pixel emission } & \multicolumn{3}{|c|}{$\begin{array}{c}\text { Scenario \#1 } \\
\left(\eta_{\mathrm{EL}}=15 \%, \eta_{\mathrm{PL}}=60 \%\right)\end{array}$} & \multicolumn{3}{|c|}{$\begin{array}{c}\text { Scenario } \# 2 \\
\left(\eta_{\mathrm{EL}}=8 \%, \eta_{\mathrm{PL}}=30 \%\right)\end{array}$} \\
\hline Parameter & $\begin{array}{c}\# \\
\text { pixel }\end{array}$ & $\begin{array}{c}\lambda_{\text {color }} \\
(\mathrm{nm})\end{array}$ & $\begin{array}{c}\mathrm{L}_{\text {color }} \\
\left(\mathrm{cd} / \mathrm{m}^{2}\right)\end{array}$ & $\begin{array}{l}\mathrm{P}_{\text {color }}^{\mathrm{W}} \\
(\mu \mathrm{W})\end{array}$ & $\begin{array}{l}\mathrm{P}_{\mathrm{B}}^{\text {color }} \\
(\mu \mathrm{W})\end{array}$ & $\begin{array}{l}\mathrm{V}_{\mathrm{LED}} \\
(\mathrm{V})\end{array}$ & $\begin{array}{l}\mathrm{I}_{\mathrm{LED}}^{\text {color }} \\
(\mu \mathrm{A})\end{array}$ & $\begin{array}{l}\mathrm{P}_{\mathrm{B}}^{\text {color }} \\
(\mu \mathrm{W})\end{array}$ & $\begin{array}{l}V_{\text {LED }} \\
(\mathrm{V})\end{array}$ & $\begin{array}{c}\mathrm{I}_{\mathrm{LED}}^{\text {color }} \\
(\mu \mathrm{A})\end{array}$ \\
\hline RED & 2 & 633 & 145,000 & 1.1 & 2.50 & 4 & 6.2 & 5.0 & 5.5 & 23.3 \\
\hline GREEN & 1 & 522 & 650,000 & 1.37 & 2.58 & 4 & 6.4 & 5.17 & 5.5 & 24.1 \\
\hline BLUE & 1 & 462 & 60,000 & 1.66 & 1.66 & 4 & 4.12 & 1.66 & 5.5 & 7.7 \\
\hline
\end{tabular}

Table I: Quad pixel specifications and operating range of micro-LED array and its active matrix to achieve $1 \mathrm{M} \mathrm{cd} / \mathrm{cm}^{2}$ assuming a high (\#1) and a low (\#2) scenario in terms of material conversion efficiencies.

\section{Design of the control IC}

Basically, the control IC ensures the interface between the video stream and the electric powering of the pixel array. For that purpose, the driving circuit combines both digital and analog cells. In the CMOS (Complementary Metal Oxide Semiconductor) driving IC used for our LED micro-display, the LED polarization circuit consists in a current source since current drive offers more accuracy for LED control due to electro-optic LED characteristic curves ( see figure $5 \mathrm{c}$ for instance).

Here, our purpose is not to thoroughly review the architecture and monitoring procedure of the active matrix, it will be done later in a dedicated publication. We will just stress some of the main design constraints to be taken into account. They specifically result either from the application specifications or from the technologies implemented in micro-display manufacturing or from both of them.

For avionics application, the user operating conditions require night as well as very bright day light environmental conditions, which means a rather wide luminance range (typ. 100 to $10^{6}$ $\mathrm{cd} / \mathrm{m}^{2}$ ) and hence wide current range available at the pixel level. This requirement will impact 
mainly the pixel control more than the pixel dimensions. This control will be done by pulse width modulation.

However, there are some constraints which will directly impact the design rules for the pixel array. As a first one, the very high luminance $\left(1 \mathrm{Mcd} / \mathrm{m}^{2}\right)$ requested together with the technologies used for micro-display manufacturing impose operating the micro-LED array in a rather high current range since micro-LED current up to $24 \mu \mathrm{A}$ under $5.5 \mathrm{~V}$ (Table I) might be necessary. This constraint will obviously condition the minimum pixel size achievable because of power density limitation. As a second constraint, the typical dispersion observed between individual micro-LED emission characteristics (see figure 5c) which will necessitate applying to each pixel permanent calibrated bias voltage to compensate for pixel-to-pixel dispersion. This constraint will make the IC design inevitably more complex. Finally, as a third constraint typical of micro-display active matrix, the need for dense power routing on active area to minimize voltage drop will also impact pixel size, hence active area size.

Taking into account all the mentioned constraints, the best compromise led us to the specifications summarized in figure 3. Compared to the data of table I, the electric performances expected for the micro-LED pixel powering ( $4 \mathrm{~V}$ maximum, $12 \mu \mathrm{A}$ mean current and $48 \mu \mathrm{W}$ mean power) is halfway between the calculated specifications of scenario \#1 and \#2. This shows that the challenge ahead will definitely deal with the search for high conversion efficiency materials, for both blue micro-LED ( $\left.\eta_{\mathrm{EL}}\right)$ and conversion layers $\left(\eta_{\mathrm{PL}}\right)$.
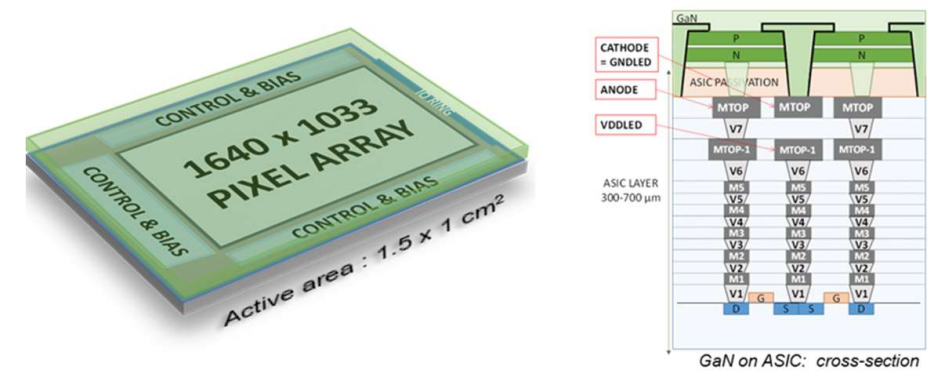

\begin{tabular}{|c|c|}
\hline Parameter & Value \\
\hline Array resolution & $1640 \times 1033$ \\
\hline Pixel pitch & $9,5 \mu \mathrm{m}$ \\
\hline Pixel mean current & $\mathbf{1 2 \mu \mathbf { A }}$ \\
\hline Max. LED polarization & $\mathbf{4 ~ V}$ \\
\hline Mean power & $\mathbf{4 8} \boldsymbol{~ W}$ \\
\hline Power consumption \\
\begin{tabular}{|r|r|} 
LLEDs \\
Digital control
\end{tabular} & $2.6 \mathrm{~W}$ \\
\hline
\end{tabular}

Figure 3: a) sketch of individual control IC developed in the HILICO project; b) sketch showing the different levels of metal lines in the IC layer and c) Main characteristics of control IC. 


\section{Micro-LED array manufacturing}

The manufacturing of micro-displays involves the transfer on the active matrix of a blue LED epitaxial layer. Until recently, this operation was done at CEA-Leti using a flip-chip technology. It required the dicing of silicon-based IC's and of GaN micro-LED arrays into individual chips, and chip to chip assembling using the so-called microtube technology ${ }^{14}$ suitable for lowtemperature hybridization of heterogeneous devices at pixel-pitch down to $10 \mu \mathrm{m}$.

Another way which should enable working with even smaller pixel pitch is the direct bonding between ICs and GaN epilayer. It is done this time at wafer level. The potential of this technology has been recently demonstrated on monochrome micro-displays ${ }^{5}$. The GaN transfer and sticking onto the active matrix is a critical technology step which requires thorough optimization in GaN surface preparation to get uniform molecular sticking on host wafer. Figure 4a depicts the principle of such transfer and sticking. It is based on metal deposition on both parts to be stuck, metal-to-metal bonding and epilayer growth substrate removal.

This approach of bonding was used to assess our ability to manufacture $10 \mu \mathrm{m}$-pitch microLED arrays. Figure $4 \mathrm{~b}$ shows an example of successful transfer and bonding of a $5 \mu \mathrm{m}$-thick, 4inch $\mathrm{GaN} / \mathrm{InGaN}$ epilayer grown on silicon onto an 8-inch Si host wafer. The epilayer growth substrate was removed by grinding. After surface cleaning, slight interference fringes are clearly visible on the transparent $\mathrm{GaN} / \mathrm{InGaN}$ layer that result from some substrate bending due to residual internal stresses in the epilayer. Such epilayer is then ready for pixel processing. Figure $4 \mathrm{c}$ depicts a typical micropixel architecture used for pixelisation test purpose. After $\mathrm{GaN} / \mathrm{InGaN}$ plasma etching to form individual $8 \mathrm{x} 8 \mu \mathrm{m}^{2}$ pixels, their electrical insulation by oxide deposition is done (in grey in figure 4c) followed by individual electrical pixel connection (in brown). In this test architecture and contrary to what happens in an active matrix, the anode 
a)

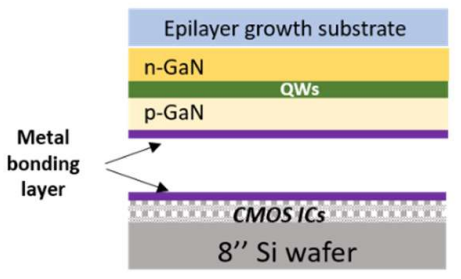

b)

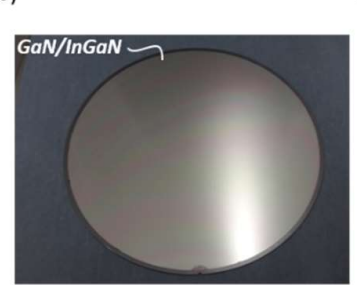

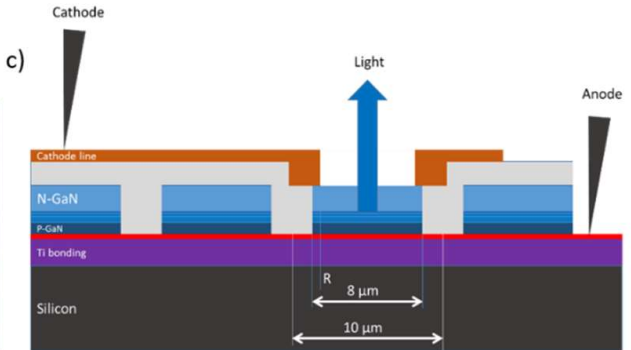

Figure 4: a) principle of GaN/InGaN epilayer transfer and bonding, b) example of GaN/InGaN epilayer uniformly transferred on an 8-inch Si host wafer, c) Typical micro-LED array test architecture used to develop the micro-display technology.

is a common electrode. Figure 5 gives electro-optical characteristics of several $10 \mu \mathrm{m}$-pitch LED pixels processed according to such test architecture. In this preliminary test the objective was more technology- than performance-oriented. That is why a very ordinary GaN/InGaN epilayer grown on silicon substrate was used. The characteristics of figure 5a corresponds to raw measurement data where the radiant power $\left(P_{B}^{W}\right)$ was measured with an angle of collection of $\pm 30^{\circ}$, which corresponds to $1 / 4$ of the total flux collected if we assume a lambertian emission. We can see in figure $5 b$ that the emission is not uniform from one pixel to another, which is not surprising with very ordinary GaN/InGaN epilayer grown on silicon. This non-uniformity in emission is confirmed by the dispersion observed in figure $5 \mathrm{c}$ where the radiant power data have been plotted versus the injected current. Note that in this graph the $\mathrm{P}_{\mathrm{B}}^{\mathrm{W}}$ data have been corrected to take into account the total emitted light flux. From this variety of characteristics, it is possible to extract the typical range of pixel current necessary to produce the few microwatts of blue light predicted by the calculation of Table I. In the most favorable case (scenario \#1), we need between 0.26 and $2.6 \mu \mathrm{W}$ of blue light per pixel to pretend reaching between 0.1 to 1 $\mathrm{Mcd} / \mathrm{m}^{2}$ of white light. In this case 50 to $330 \mu \mathrm{A}$ per pixel under a bias voltage of 6-8 V should be necessary, which clearly exceeds the ASIC capacity. This is because of the very poor external conversion efficiency of the epilayer used for this test. 

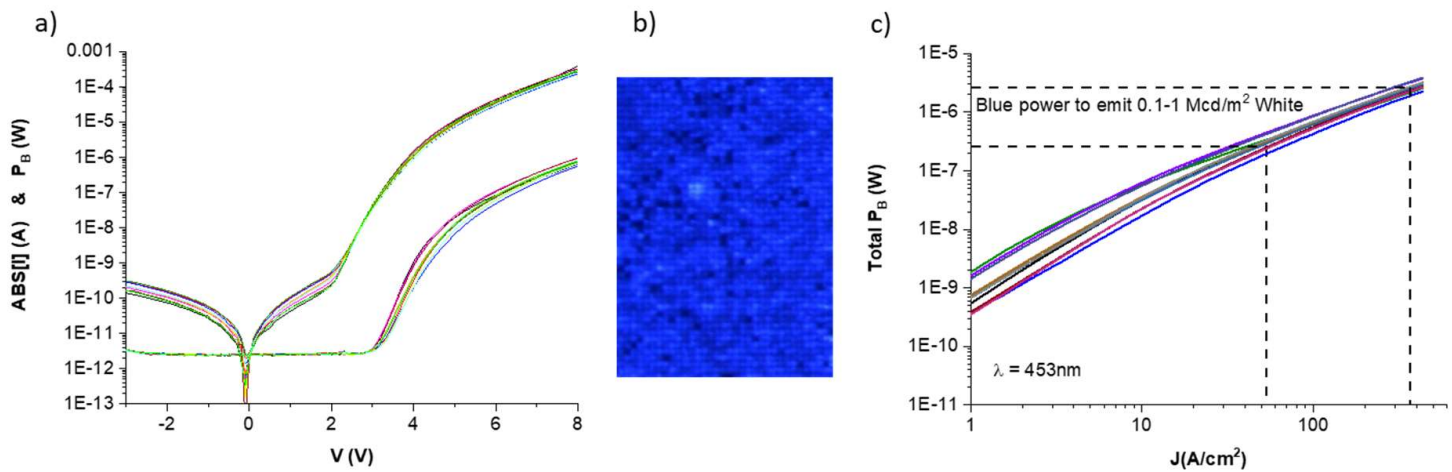

Figure 5: a) I-V and $P_{B}^{W}-V$ raw characteristics of 10 individual pixels; $I$ is the current per pixel; b) optical microscope picture showing the emitting surface of an array of hundreds of pixels with all pixel lighted-on; c) $P_{B}^{W}$-J curves deduced from figure 5 a and corrected by a factor 4 to get the total emitted radiant power. $J$ is the current density.

We estimate that in the present case $\eta_{E L} \sim 0.5 \%$, at least one order of magnitude lower than necessary. As a result the experimental current deduced from figure $5 \mathrm{c}$ is at least one order of magnitude too high.

In $\mathrm{GaN} / \mathrm{InGaN}$ micro-LED arrays, the origin of such weak electroluminescence conversion efficiency is well known and mainly comes from intrinsic epilayer defects (density of dislocations $>10^{8} \mathrm{~cm}^{-2}$ ) or extrinsic ones (etching-induced pixel wall defects, difficult p-contact hole injection, ...). By working on all these parameters, it is possible to improve the $\eta_{E L}$ factor to a level compatible with the requirements of table I. Figure 6 shows an example of material development done within the HILICO project with the corresponding V-J and $\eta_{\mathrm{EL}^{-}}-\mathrm{J}$ characteristics reached so far on $80 \mu \mathrm{m}^{2}$ square micro-LEDs . In the photometric test conditions used in this work, part of the light is lost in the sapphire substrate because of light guiding caused by total internal reflection. Despite this, $\eta_{E L}$ reaches nearly $9 \%$, in agreement with targeted specification (scenario \#2). Even more interesting is the possibility to tune the epilayer stack so that the nominal ASIC operating range perfectly matches with the maximum of $\eta_{\mathrm{EL}^{-}}-\mathrm{J}$ curve. As a result, in such micro-LED array, it is possible to inject up to $12 \mu \mathrm{A}$ per pixel (15 $\mathrm{A} / \mathrm{cm}^{2}$ ) under a voltage of $3 \mathrm{~V}$, namely $48 \mu \mathrm{W}$ electric power which can be converted with an 
a)

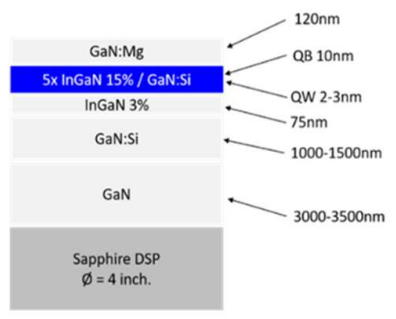

b)

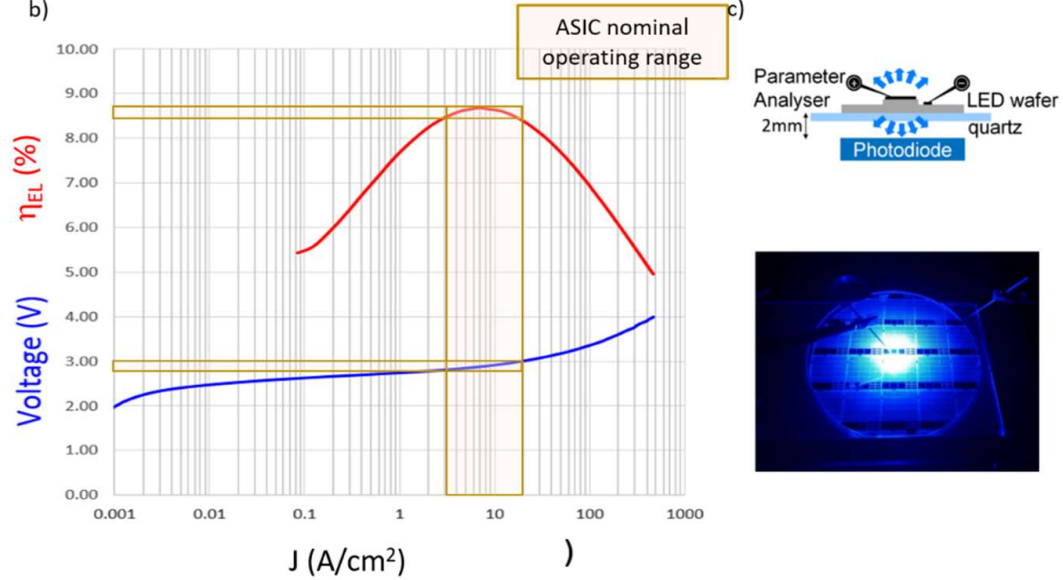

Figure 6: a) epilayer stack; b) typical $V-J$ and $\eta_{E L^{-}}{ }^{-}$characteristics of an individual pixel (square pixel: $80 \mathrm{\mu m}^{2}$ ) processed on an optimized GaN/InGaN epilayer; c) view of testing bench with testing conditions (upper sketch).

efficiency of $8 \%$ minimum. We can compare this situation with the specifications given in table I for scenario \#2. In this case, assuming a color conversion efficiency $\eta_{\mathrm{PL}}=30 \%$, the production of $1 \mathrm{Mcd} / \mathrm{m}^{2}$ requires for the green and red pixel to inject an electric power up to $130 \mu \mathrm{W}$. Hence, we can estimate that with the micro-LED array demonstrated in this work, with $48 \mu \mathrm{W}$, we could expect reaching $\sim 0.35 \mathrm{Mcd} / \mathrm{cm}^{2}$, provided $\eta_{\mathrm{PL} \geq} 30 \%$. The ability of meeting this last specification is discussed hereafter according to the color conversion technology to be considered.

\section{Color conversion technological routes}

We have identified two potential technologies which may be used for the present high luminance application. The first route is based on semiconductor nanocrystals synthesized by solution chemistry (so-called Quantum Dots (QDs)). The second route uses quantum wells (QWs) grown by vacuum epitaxy.

\section{a. Quantum Dots}

To our knowledge, the most efficient QDs used so far for light conversion in the visible range are based on cadmium and zinc chalcogenides (CdSe, $\mathrm{CdS}, \mathrm{ZnS}, \mathrm{ZnSe})$. Their interest for 
display application is their narrow emission spectral bandwidth (Full Width Half Maximum, FWHM= 40-60 nm). With this respect, using nanoplatelets (NPL) instead of nanocrystals has been shown to decrease even further this FWHM bandwidth. The successful synthesis of fluorescent colloidal CdSe NPLs demonstrated 10 years ago ${ }^{15}$, showed emission FWHM ranging from 20 to $30 \mathrm{~nm}$, paving the way to RGB displays with purer color primaries. However, for very bright full color micro-display application, these NPLs must withstand very high blue radiant power (up to $6 \mathrm{~W} / \mathrm{cm}^{2}$ ), which necessitates dedicated chemical processes to stabilize them.

We did preliminary experiments to integrate red-emitting CdSe-based NPLs onto blue microLEDs. Synthesis of various kinds of CdSe-based NPLs was done involving similar CdSe core NPLs but different shell layers. Figure 7a shows an example of red-emitting CdSe-based NPLs synthesized in a significant quantity. Figure $7 \mathrm{~b}$ demonstrates how the different shells can drastically mitigate the photoluminescence (PL) efficiency decay under ageing. The tests have been intentionally done in very harsh conditions $\left(30 \mathrm{~W} / \mathrm{cm}^{2}\right.$ blue light continuous exposure) to accelerate the ageing kinetics. Initially, all the NPLs exhibit, in solution, an internal conversion efficiency of at least $50 \%$. Under ageing test they however behave very differently. While CdSe/ZnS NPLs exhibit a relatively severe PL decay, substitution of ZnS by CdS shell seems to start mitigating this ageing PL decay. In a more complex chemical approach, using a double shell combining $\mathrm{CdS}$ and $\mathrm{ZnS}$ prove to be particularly efficient and provides a rather outstanding PL stabilization. This route seems to be definitely promising.

Another key issue to be assessed for LED micro-display application is the ability to pattern the NPL-resist composite material that must be used to deposit the QDs in a controlled manner. For that purpose, a proprietary resist was adapted to be mixed with CdSe-based NPLs. As preliminary test, the NPL-containing resist was spin-coated on glass substrates, exposed to iline photolithography and stripped. Fig.7c shows that NPL patterns with pixel/pitch as low as 
a)

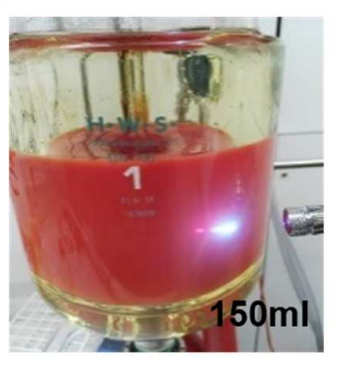

b)

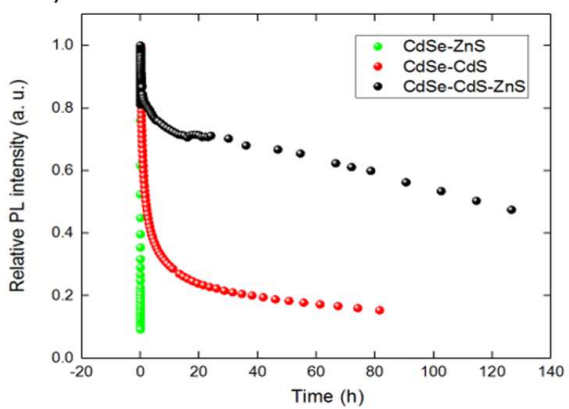

c)

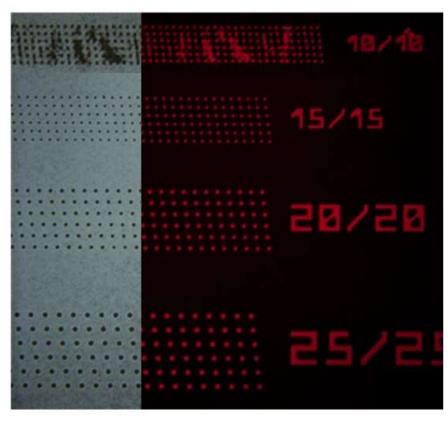

Figure 7: a) Red NPL synthesis; b) Photoluminescence (PL) ageing test on the red NPL of different compositions under continuous blue light exposure $\left.\left(\lambda=450 \mathrm{~nm}, P=30 \mathrm{~W} / \mathrm{cm}^{2}\right) ; c\right)$ first patterning tests on glass showing well resolved NPL patterns of size down to a pixel/pitch of 10/10 $\mu$ m - optical microscope image: under white light illumination (left) and under blue illumination with a red filter (right).

$10 / 10 \mu \mathrm{m}$ can be resolved, providing colored pixel sizes in line with our application requirement. Also interesting is that, after lithography and stripping, the NPLs are still active under blue light as shown by Fig 7c (right) where clear red emission by the small dots is visible. Further characterization of such NPL route is still ongoing to evaluate its conversion efficiency and photo-stability after patterning.

\section{b. Quantum Wells}

As a second light conversion technology, epilayers with dedicated QWs can also be considered. We expect for this alternative route even better photo-stability under very high blue flux. However, many issues have still to be solved. Among them, the implementation of an efficient mechanical and optical coupling between micro-LED array and conversion epilayers is a prerequisite. The accurate assessment of color conversion efficiency $\left(\eta_{P L}\right)$ is another key issue, since light extraction from this conversion epilayers is not easy. They both exhibit rather high refractive index $\left(\mathrm{n}_{\mathrm{GaN}}=2.5\right.$ and $\left.\mathrm{n}_{\mathrm{AlInGaP}}=3.5\right)$ promoting light guiding and trapping.

The integration of conversion epilayers could be done on the same technological basis as used for bonding the LED epilayer to the Si-based IC. However, in this case, oxide-mediated direct bonding must be developed. Such a technological step has been demonstrated on a test sapphire 

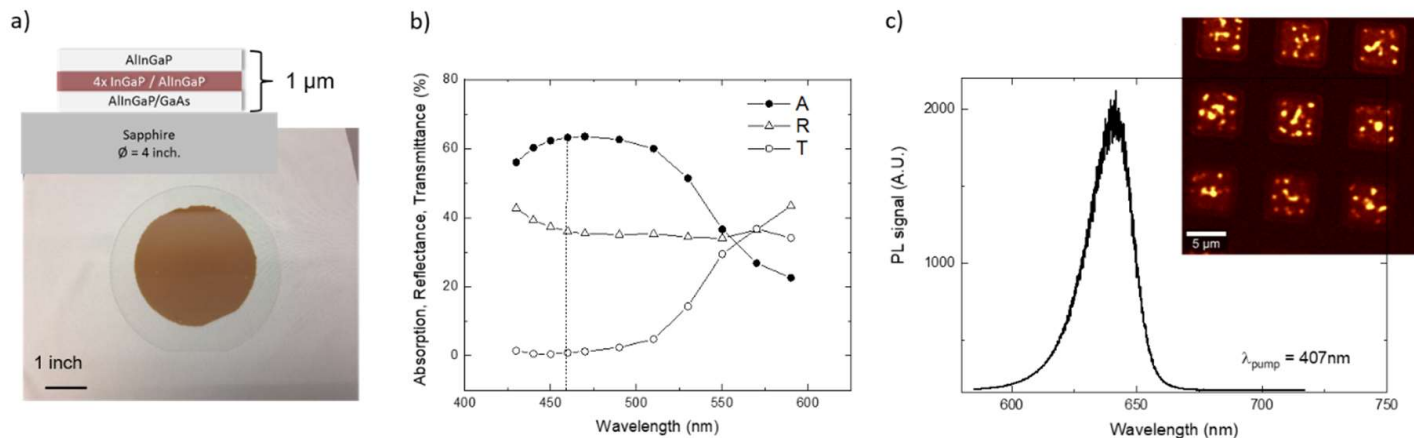

Figure 8: a) Picture of a 3-inch AInGaP/InGaP $Q W$ layer transferred on a transparent sapphire substrate. Upper left, the corresponding red-conversion epilayer stack; b) Spectral optical absorption $(A)$, reflection $(R)$ and transmission $(T)$ of red-conversion layer and c) Typical photoluminescence $P L$ signal at 640nm measured on same pixelated layer. The laser pump is in deep blue with radiant power 2 or 3 orders of magnitude higher than in our application.

sample. A $1 \mu \mathrm{m}$-thick 3-inch AlInGaP/InGaP-QW epilayer grown on GaAs substrate has been thus successfully bonded to a sapphire substrate for further optical characterization (figure 8a). After removal of GaAs substrate by grinding and further CMP polishing (Chemical Mechanical Planarization), the surface of the red-conversion epilayer appears particularly smooth, demonstrating uniform adhesion. Optical characterization of such thin layer shows high absorption $(63 \%)$ and no transmission of blue light $(\lambda=460 \mathrm{~nm})$, characteristics particularly interesting for our application. However, despite evidences of red photoluminescence with an interesting low FWHM (25 nm), red light extraction is difficult (red light is only visible on pixel surface irregularities - see figure 7c) and will require further developments.

As for green-conversion layer, the epilayer is based on GaN/InGaN-QW stacks tuned for green emission and containing multiple QWs. Figure 9a shows the TEM (Transmission Electronic Microscopy) microstructure cross-section of an epilayer grown specifically for light conversion (25 QWs). Figure 9b gives the spectral absorption measured on several epilayer stacks. Considering that those QW structures incorporate between 25 and 35 QWs we estimate an average blue absorption $(\lambda=440 \mathrm{~nm})$ of $1.07 \%$ per QW. Hence, complete absorption of the blue 
emission at $440 \mathrm{~nm}$ would require around 94 QWs, which is not feasible without degradation of the epilayer crystalline structure. At the pixel level, integration strategies are thus required to recycle several times the blue pumping light. Figure 9c shows a typical photoluminescence spectrum of such green-conversion epilayers. The emission FWHM is much larger $(50 \mathrm{~nm})$ than the bandwidth measured for structures incorporating only 5 QWs. This indicates a QWindium concentration drift throughout the epilayer.

a)

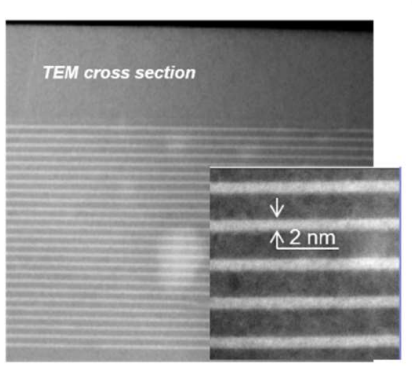

b)

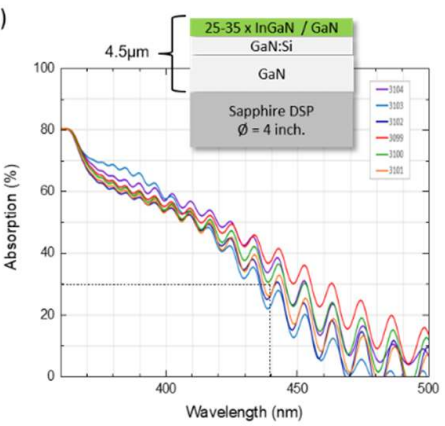

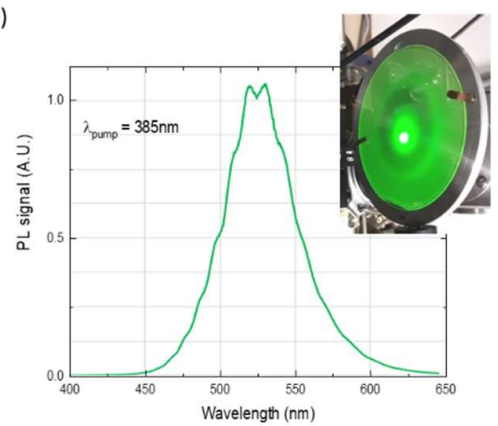

Figure 9: a) Typical TEM cross-section of green-conversion epilayer showing the stacking of 25 InGaN $Q W s$. The thickness of each $Q W$ is around $2 \mathrm{~nm}$; b) Spectral optical absorption of various green conversion epilayers comprising between 25 and 35 QWs; c) Typical photoluminescence PL signal measured on same layers. The optical pumping uses UV laser. The picture shows a 4-inch green conversion epilayer under test.

\section{c. Summary}

Table II highlights the main features of both conversion routes studied in this work.

\begin{tabular}{|c|c|c|c|c|c|}
\hline Route & $\begin{array}{l}\text { Integration } \\
\text { technology }\end{array}$ & Feasibility & Blue absorption & $\begin{array}{l}\text { Conversion } \\
\text { efficiency }\end{array}$ & Photostability \\
\hline QD-red & $\begin{array}{c}\text { Spin-coating + } \\
\text { pixelisation (litho.) }\end{array}$ & $\begin{array}{c}\text { Color pixel } \\
\text { demonstrated }\end{array}$ & $\begin{array}{c}\text { moderate } \\
\text { (thickness limited } \\
\text { at } 10 \mu \mathrm{m})\end{array}$ & $\begin{array}{c}\text { QDs: } 50 \% \\
\text { QDs/resist*: } 30 \%\end{array}$ & $\begin{array}{l}\text { Promising } \\
\text { Chemistry } \\
\text { developed }\end{array}$ \\
\hline QW-red & \multirow{2}{*}{$\begin{array}{l}\text { Transfer of epilayers } \\
+ \text { pixelisation (litho.) }\end{array}$} & \multirow{2}{*}{$\begin{array}{c}\text { Transfer } \\
\text { demonstrated }\end{array}$} & Very high & Not measured yet & \multirow{2}{*}{$\begin{array}{c}\text { Should be stable by } \\
\text { nature } \\
\text { (epilayer) }\end{array}$} \\
\hline QW-green & & & $\begin{array}{c}\text { Low (Number of } \\
\text { QW limited) }\end{array}$ & Not measured yet & \\
\hline
\end{tabular}

*Study on-going not presented here

Table II: Main characteristics of conversion technologies studied in this work. 


\section{Conclusion}

Starting from the specifications of the display to be used in an avionics head-up display, an approach to specify the various building blocks has been proposed and detailed. On this basis, the specifications of the main technology building blocks which constitute the full color LED micro-display and entering into the manufacturing process flow have been deduced. The specifications have been then compared to our technology capabilities. As a result, this dimensioning study has highlighted some interesting results:

○ In the best material scenario $\left(\eta_{\mathrm{EL}}=15 \%, \eta_{\mathrm{PL}}=60 \%\right)$, the achievement of a $1 \mathrm{M} \mathrm{cd} / \mathrm{m}^{2} \mathrm{RGB}$ LED micro-display requires an active matrix providing $91.7 \mu \mathrm{W}(4 \mathrm{~V} / 22.9 \mu \mathrm{A}$ - see table I) per RRGB QUAD pixel. With the chosen active matrix design ( $1 / 4[1640 \times 1033]$ QUAD pixels) and the available electric power $(2.6 \mathrm{~W})$, up to $6.7 \%$ of total number of pixels could emit at maximum luminance. This is acceptable for symbology applications, not for video where a ratio of $\sim 20 \%$ is necessary. However, using such micro-displays in video mode is still possible by limiting to $300,000 \mathrm{~cd} / \mathrm{m}^{2}$ the need in micro-display luminance through the design of a combiner providing a lower expansion factor $(\mathrm{EF}=3)$ and integrating more transparent optics (higher efficiency $\eta$ ) .

$\circ$ With more realistic material performances $\left(\eta_{\mathrm{EL}}=8 \%, \eta_{\mathrm{PL}}=30 \%\right)$ still interesting luminance $\left(0.35 \mathrm{Mcd} / \mathrm{m}^{2}\right)$ are reachable with however restrained imaging possibilities. With a maximum power of $4 \times 48=192 \mu \mathrm{W}$ delivered to each RRGB QUAD pixel (to reach 0.35 $\mathrm{Mcd} / \mathrm{m}^{2}$ ), only $3.2 \%$ of total number of pixels could indeed emit at maximum luminance. Operating such micro-displays in video mode imposes using a simple combiner without pupil expansion $(\mathrm{EF}=1)$, which could however necessitate more complex and bulky optics.

$\circ$ With the present maturity of GaN micro-LED array technology there is still significant pixel-to-pixel emission dispersion requiring individual pixel correction at the display level and hence more complex active matrix design. Such issue must be better addressed in the 
micro-LED technology roadmap in addition to the search for maximum electroluminescence external quantum efficiency.

- Two color conversion routes have been explored. The NPL route has proven to provide conversion materials offering interesting initial $\eta_{\mathrm{EL}}$, improved photo-stability and promising integration ability. The key issues still remain the level of conversion efficiency and the photo-stability under a blue radiant power of a few $\mathrm{W} / \mathrm{cm}^{2}$ once integrated on a micro-LED array via resist-NPL compound integration. With this respect, the 2D-epilayer approach could be an interesting and credible alternative but its implementation is still in its infancy, requiring much further investigation.

Acknowledgements: The authors acknowledge funding from the CleanSky-H2020 HILICO European project (under H2020-EU.3.4.5.6. - ITD Systems, Project ID: 755497). We also acknowledge J.L. Thomassin for his valuable technical contribution to the micro-LED technology.

\section{References:}

${ }^{1}$ G. Haas, "Microdisplays for Augmented and Virtual Reality," SID 2018 Symposium Digest of Technical Papers, p. 506, 201.

${ }^{2}$ https://www.displaydaily.com/article/display-daily/kopin-brillian-lcd-microdisplay-to-be-inarmy-ar-hmd.

3 J. Day et al., "III-nitride full-scale high-resolution micro-displays," Appl. Phys. Lett., 99, 031116 (2011).

${ }^{4}$ F. Templier et al., "GaN-based emissive micro-displays: a very promising technology for compact, ultra-high brightness display systems," SID Digest of Technical Papers, 1013-1016 (2016).

${ }^{5}$ L. Zhang et al, "Wafer-scale monolithic hybrid integration of Si-based IC and III-V epilayers A mass manufacturable approach for active matrix micro-LED micro-displays", Journal of the SID 26/3, 2018 
${ }^{6}$ F. Templier et al, « High-resolution, active-matrix, 10- $\mu$ m pixel-pitch GaN LED microdisplays for Augmented Reality applications”, SPIE Proc. Vol. 10556, (2018); https://doi.org/10.1117/12.2294527.

${ }^{7}$ http://www.plesseysemiconductors.com/products/microleds/

${ }^{8}$ https://optics.org/news/10/12/11 , plessey communication, 06-12-2019.

${ }^{9}$ Hyo-Min Kim et al., J Soc Inf Display. 2019; 1-7. J. of the SID, “ Ten micrometer pixel, quantum dots color conversion layer for high resolution and full color active matrix microLED display", https://doi.org/10.1002/jsid.782

${ }^{10}$ J. Osinski et al., "Quantum Dot Design Criteria for Color Conversion in Micro-LED Displays", SID 2019 DIGEST, 34-37.

${ }^{11}$ F. Gou et al; "High performance color-converted micro-LED displays"; J Soc Inf Display. 2019;27:199-206., DOI: 10.1002/jsid.764

${ }^{12}$ Clean Sky's HiLiCo: Bright ideas take flight [Online]. Available: https://www.cleansky.eu/clean-skys-hilico-bright-ideas-take-flight

${ }^{13}$ F. Templier et al., "Blue and Green 10- $\mu \mathrm{m}$ pixel pitch GaN LED Arrays with very high brightness", The 22nd International Display Workshops 2015 (IDW'15).

${ }^{14}$ H B. Goubault de Brugière et al., "A 10 $\mu \mathrm{m}$ Pitch Interconnection Technology using Micro Tube Insertion into Al-Cu for 3D Applications", Electronic Components and Technology Conference, IEEE (2011).

${ }^{15}$ S. Ithurria et al., J. AM. CHEM. SOC. 2008, 130, 16504-16505 